\title{
On Peamo's theorem in locally convex spaces
}

by

\author{
KARI ASTALA (Helsinki)
}

Abstract. In this paper we consider ordinary differential equations in locally convex spaces. We show that Peano's theorem holds in the spaces $E=\left(X^{\prime}, \tau\right)$, where $X^{\prime}$ is the dual of a barrelled normed space $X$ and $\tau$ is any locally convex vector topology of $X^{\prime}$ that is stronger than the $w^{*}$-topology but weaker than the topology of precompact convergence; $\sigma\left(X^{\prime}, X\right) \lesssim \tau \lesssim \lambda\left(X^{\prime}, X\right)$. Such spaces $E$ are the only sequentially complete locally convex spaces that contain a compact barrel.

On the other hand, we prove that Peano's theorem does not hold in non-semireflexive quasi-complete spaces or in nonreflexive Banach spaces equipped with the weak topology. In particular, this yields a new characterization for reflexivity in Banach spaces: A Banach space $E$ is reflexive if and only if Peano's theorem holds in the space $\left(E, \sigma\left(E, E^{\prime}\right)\right)$.

1. Introduction. In this paper we study ordinary differential equations defined in a real locally convex vector space. We present some answers to the question:

(1) Which locally convex spaces have the Peano property?

1.1. Definimion. Let $E$ be a topological vector space. We say that $E$ has the Peano property (or that Peano's theorem holds in $E$ ) if for each continuous mapping $f: \boldsymbol{R} \times \boldsymbol{E} \rightarrow E$ and for each point $\left(t_{0}, x_{0}\right) \in \boldsymbol{R} \times E$ the Cauchy problem

$$
x^{\prime}(t)=f(t, x(t)), \quad x\left(t_{0}\right)=x_{0},
$$

has a solution defined in some neighbourhood of $t_{0}$.

We shall mostly consider sequentially complete spaces; only to such spaces can integral calculus be applied.

Some results concerning problem (1) are already known. For instance, Godunor [4] has proved that a Banach space $E$ has the Peano property if and only if $E$ is finite-dimensional. On the other hand, in [11] Szép showed that Peano's theorem holds in every reflexive Banach space equipped with the weak topology.

After the preliminaries of Chapter 2 we shall prove that if a sequentially complete locally convex space $E$ satisfies the condition

(2) $E$ contains a compact barrel, 
then $E$ has the Peano property. This result contains the theorem of Szép as a special case. In fact, we shall see that $E$ is a sequentially complete locally convex space satisfying (2) if and only if

$$
E=\left(X^{\prime}, \tau\right),
$$

where $X^{\prime}$ is the dual of a barrelled normed space $X$ and $\tau$ is any topology of $X^{\prime}$, which is stronger than the $w^{*}$-topology but weaker than the topology of precompact convergence; briefly

$$
\sigma\left(X^{\prime}, X\right) \lesssim \tau \lesssim \lambda\left(X^{\prime}, X\right)
$$

It remains open, whether there exists a sequentially complete locally convex space $E$, which has the Peano property but not property (2).

Finally, in Chapter 4 we generalize the construction of Cellina [1] and show that a quasi-complete locally convex space $E$ must be semireflexive if it has the Peano property. We also prove the converse to Szép's theorem and thus find a new characterization for reflexivity in Banach spaces: A Banach space $E$ is reflexive if and only if $\left(E, \sigma\left(E, E^{\prime}\right)\right)$ has the Peano property. This result was conjectured in [2].

\section{Preliminaries.}

Notatrons. We shall always assume that the topological vector spaces considered are Hausdorff spaces and that their scalar field is $\boldsymbol{R}$.

We use the notations of Horvath [7], to which we refer for the basic concepts of topological vector spaces. Especially, if $E$ is a locally convex space, $\sigma\left(E^{\prime}, E\right)$ denotes the $w^{*}$-topology, $\lambda\left(\mathbb{E}^{\prime}, E\right)$ the topology of precompact convergence, and $\beta\left(E^{\prime}, E\right)$ the strong topology of $E^{\prime}$.

Further, $\overline{c o} B$ stands for the closed convex hull of a subset $B \subset E$ and $C([a, b], E)$ for the space of all continuous functions $f:[a, b] \rightarrow E$.

The Riemann integral. For completeness we recall the basic propertios of the Riemann integral. Let $D=\left\{t_{i}\right\}_{i=0}^{n}$ be a partition of $[a, b] \subset \boldsymbol{R}$ (that is $a=t_{0}<t_{1}<\ldots<t_{n}=b$ ). The norm of $D$ is $\|D\|=\max _{1 \leqslant i \leqslant n}\left\{\mid t_{i}-\right.$

2.1. Definrtron. Let $E$ be a locally convex vectior spaco. A mapping $f:[a, b] \rightarrow E$ is (Riemann) integrablc if thero is a vector $\int_{a}^{b} f(t) d t \in E$ such
that

$$
\lim _{\|D\| \rightarrow 0} \sum_{k=1}^{n} f\left(s_{k}\right)\left(t_{k}-t_{t-1}\right)=\int_{a}^{b} f(t) d t
$$

in the topology of $E$. Here $D=\left\{t_{k}\right\}_{k=0}^{n}$ and $s_{k} \in\left[t_{k_{0}-1}, t_{\psi_{k}}\right]$ is arbitrary.

The following facts are easily established:

(3.a) If $f \in O([a, b], E)$ and $\overline{c o}\{f[a, b]\}$ is sequentially complete, then $f$ is integrable. (3.b) $\quad \int_{a}^{b} f(t) d t \in(b-a) \overline{\mathrm{co}}\{f[a, b]\}$.

(3.c) If $f$ is continuous and integrable and $F(t)=\int_{a}^{t} f(s) d s, a \leqslant t \leqslant b$, then $F^{\prime}(t)=f(t)$ for each $t \in[a, b]$.

It is not difficult to construct a non-sequentially complete locally convex vector space $E$ and a continuous $g:[a, b] \rightarrow E$ that is not integrable. Such a mapping $g$ has no primitive, i.e. a differentiable mapping $x:[a, b] \rightarrow$ $\rightarrow E$ such that

$$
x^{\prime}(t)=g(t) .
$$

Hence in spaces $E$ that are not sequentially complete there can exist this kind of trivial counterexamples to Peano's theorem. In other words, sequentially complete spaces is the appropriate class of locally convex spaces for the study of the Peano property.

3. Existence theorems. The standard proofs of the classical Peano's theorem cannot be generalized as such to an infinite-dimensional vector space since they are based on the local compactness of $\boldsymbol{R}^{n}$ (see e.g. [6], p. 10). However, the ideas of these proofs can be applied in spaces that contain "large" compact sets.

The following form of the Banach-Mackey theorem will be usefull. For a proof see [9], p. 91.

3.1. THEOREM (Banach, Mackey). Suppose $E$ is locally convex, $B \subset E$ a barrel and $K$ a closed, convex, bounded, balanced and sequentially complete subset of $E$. Then $B$ absorbs $K$.

3.2. THEoRem. Suppose $E$ is locally convex, $B \subset E$ a barrel, $t_{0} \in[a, b)$ $\subset \boldsymbol{R}, x_{0} \in E$ and let $f: \boldsymbol{R} \times E \rightarrow E$ be any mapping. Denote by $S$ the set $\mathbb{S}$ $=[a, b] \times\left(x_{0}+B\right) \subset \boldsymbol{R} \times E$.

If $\left.f\right|_{S}$ is continuous and $\overline{\mathrm{co}}\{f(S)\}$ is compact, the differential equation

$$
x^{\prime}(t)=f(t, x(t)), \quad x\left(t_{0}\right)=x_{0}
$$

has a solution defined on $\left[t_{0}, t_{0}+\delta\right]$ for some $\delta>0$.

Proof. As the balanced hull of a convex compact set is convex. and compact, $B$ absorbs $\overline{c o}\{f(S)\}$ by Theorem 3.1. So we can choose a $\delta>0$ such that $\left[t_{0}, t_{0}+\delta\right] \subset[a, b]$ and $\delta \overline{c o}\{f(S)\} \subset B$.

For brevity we denote $I=\left[t_{0}, t_{0}+\delta\right]$. Let $\varepsilon$ be any real number such that $0<\varepsilon \leqslant \delta$. Set

$$
u_{\varepsilon}(t)= \begin{cases}x_{0}, & 0 \leqslant t-t_{0} \leqslant \varepsilon \\ x_{0}+\int_{t_{0}}^{t-\varepsilon} f\left(s, u_{s}(s)\right) d s, & \varepsilon \leqslant t-t_{0} \leqslant \delta .\end{cases}
$$


Then $u_{\varepsilon}: I \rightarrow E$ is well definied and continuous. The integral in (4) exists by (3.a). According to formula (3.b) each $u_{s}$ satisfies the conditions

$$
\begin{aligned}
& u_{s}\left(t_{0}\right)=x_{0}, \\
& u_{\varepsilon}(t) \in x_{0}+\left(t-\varepsilon-t_{0}\right) \overline{c 0}\{f(S)\} \subset x_{0}+B \quad \forall t \in I, \\
& u_{s}(t)-u_{s}(s) \in(t-s) \overline{\operatorname{co}}\{f(S)\} \quad \forall s, t \in I .
\end{aligned}
$$

Let $\delta=\varepsilon(1)>\varepsilon(2)>\varepsilon(3)>\ldots>0$ be a decreasing sequence with $\lim \varepsilon(n)=0$. As $\overline{\operatorname{co}}\{f(S)\}$ is compact, the family of continuous mappings $\stackrel{n \rightarrow \infty}{H}=$

$=\left\{u_{e(n)}\right\}_{n=1}^{\infty}$ is equicontinuous and the set $H(t)=\left\{u_{s(n)}(t)\right\}_{n=1}^{\infty}$ is relatively compact in $E$ for every $t \in I$. Now, according to Ascoli's theorem ([9], p. 81), $H$ is relatively compact in the space $C(I, E)$ if we equip $O(T, E)$ with the topology of uniform convergence.

Let $u \in C(I, E)$ be any cluster point of the set $H$. Then the continuous mapping $t \rightarrow f(t, u(t)), t \in I$, is integrable. We claim that

$$
u(t)=x_{0}+\int_{t_{0}}^{t} f(s, u(s)) d s \quad \forall t \in I .
$$

Suppose $U \subset D$ is a barrelled neighbourhood of $\overline{0}$. If $D$ denotes the balanced hull of $\overline{\operatorname{co}}\{f(S)\}$, the set $G=I \times\left(x_{0}+\delta D\right)$ is compact and hence the restriction of $f$ to $G$ is uniformly continuous. Choose a barrelled neighbourhood $V$ of $\overline{0}$ such that $f(s, x)-f(s, y) \in U$, whenever $x-y \in V$ and $(s, x),(s, y) \in G$.

There exists a natural number $n \in \boldsymbol{N}$ satisfying $\varepsilon(n) \overline{\operatorname{co}}\{f(S)\} \subset U$ and $u(s)-u_{e(n)}(s) \in V \cap U$ for every $s \in I$. Then, by the above facts,

$$
\begin{aligned}
& u(t)-x_{0}-\int_{t_{0}}^{t} f(s, u(s)) d s \\
& =u(t)-u_{\varepsilon(n)}(t)+\int_{t_{0}}^{t}\left[f\left(s, u_{e(n)}(s)\right)-f(s, u(s))\right] d s-\int_{t-s(n)}^{t} f\left(s, u_{e(n)}(s)\right) d s \\
& \in U+\left(t-t_{0}\right) U-\varepsilon(n) \overline{c o}\{f(S)\} \subset(2+\delta) U .
\end{aligned}
$$

As $U$ is arbitrary, equation (6) is proved. Finally, the theorem follows from (6) and from (3.c).

Remark. It is possible to show that in the hypotheses of Theorem 3.2 the compactness of $\overline{c o}\{f(S)\}$ can be replaced by the sequentially compactness of $\overline{\mathrm{co}}\{f(S)\}$.

Assume that the locally convex space $E$ is sequentially complete and that $E$ contains a compact barrel. Then, by Theorem 3.1, the closed and bounded subsets of $E$ are compact. Moreover, every continuous mapping $f: \boldsymbol{R} \times E \rightarrow E$ satisfies the hypotheses of Theorem 3.2. Consequently, $E$ has the Peano property.
3.3. LEMma. Let $E$ be a topological vector space. Then $E$ is a sequentially complete locally convex space containing a compact barrel if and onty if

$$
E=\left(X^{\prime}, \tau\right),
$$

where $X^{\prime}$ is the dual of a barrelled normed space $X$ and the locally convex topology $\tau$ of $X^{\prime}$ satisfies

$$
\sigma\left(X^{\prime}, X\right) \lesssim \tau \lesssim \lambda\left(X^{\prime}, X\right) .
$$

Proof. Suppose $X^{\prime}$ is the dual of a normed and barrelled space $\boldsymbol{X}$ and $\tau$ a topology of $X^{\prime}$ satisfying (7). Denote by $B_{1}$ the closed unit ball of $X^{\prime}, B_{1}=\left\{x^{\prime} \in X^{\prime} \mid\left\|x^{\prime}\right\| \leqslant 1\right\}$. By the theorems of Banach-Alaoglu ([7], p. 201) and Banach-Dieudonné ([7], p. 245) $B_{1}$ is compact in the space $\left(X^{\prime}, \lambda\left(X^{\prime}, X\right)\right)$. Thus $B_{1}$ must be compact also in the space $\left(X^{\prime}, \tau\right)$. Obviously $B_{1}$ is a barrel in $\left(X^{\prime}, \tau\right)$.

Let $K \subset X^{\prime}$ be bounded in the $\tau$-topology. Then $K$ is $\sigma\left(X^{\prime}, X\right)$ bounded and so, $X$ being barrelled, it is norm bounded ([7], p. 212). As $K \subset r B_{1}$ for some $r \in \boldsymbol{R}, K$ must be $\tau$-relatively compact. Hence $\left(X^{\prime}, \tau\right)$ is sequentially complete; the space $\left(X^{\prime}, \tau\right)$ is, in fact, quasi-complete.

Conversely, suppose $E$ is a sequentially complete locally convex space, which contains a compact barrel $B$. We shall denote the topology of $E$ by $\tau_{0}$. According to Theorem 3.1 $B$ absorbs all the bounded sets of $E$. So each closed and bounded subset of $E$ must be compact. In particular, $E$ is semireflexive (cf. [7], p. 227).

For the space $X$ we choose

$$
X=\left(E^{\prime}, \beta\left(E^{\prime}, E\right)\right) .
$$

As $B$ absorbs all the bounded sets of $E$, its polar $B^{\circ}$ is a bounded neighbourhood of $\overline{0}$ in $X$; in other words, $X$ is normable. Now $X^{\prime}=\left(E^{\prime \prime}, \beta\left(E^{\prime \prime}, E^{\prime}\right)\right.$ is a Banach space and from the semireflexivity of $E$ we deduce

$$
\left(E, \tau_{0}\right)=\left(X^{\prime}, \tau\right),
$$

where the topology $\tau$ is weaker than the norm topology of $X^{\prime}$.

Wo shall show that $\tau$ satisfies (7). First, $\tau$ is stronger than $\sigma\left(X^{\prime}, X\right)$ since $X$ is the dual of $\left(X^{\prime}, \tau\right)=\left(E, \tau_{0}\right)$. Next, using the theorem of BanachDieudonne ([7], p. 245) we see that $\lambda\left(X^{\prime}, X\right)$ is the strongest of those topologies of $X^{\prime}$ that induce the same topology as $\sigma\left(X^{\prime}, X\right)$ on the bipolar $B^{\circ 0}=B$. Therefore we must prove that the restriction of the identity mapping id: $\left(X^{\prime}, \tau\right) \rightarrow\left(X^{\prime}, \sigma\left(X^{\prime}, X\right)\right)$ to the set $B$ is a homeomorphism. But by the above id $\left.\right|_{B}$ is a continuous bijection and as $B$ is compact, id $\left.\right|_{B}$ is, in fact, a homeomorphism.

It remains to show that $X$ is barrelled. From the fact $\left(X^{\prime}, \tau\right)^{\prime}=X$ we see that every $\sigma\left(X^{\prime}, X\right)$-bounded subset of $X^{\prime}$ is $\tau$-bounded. Moreover, the sequentially completeness of $\left(X^{\prime}, \tau\right)$ together with Theorem 3.1 imply 
that $\tau$-bounded sets are absorbed by $B$; that is, $\tau$-bounded sets are norm bounded. Therefore strong and $\sigma\left(X^{\prime}, X\right)$-boundedness coincide in $X^{\prime}$, which proves the last of our assertions ([9], p. 171).

It is well known that every Banach space is barrelled. Note, however, that there are noncomplete barrelled and normed spaces (see e.g. [5], p. 109).

3.4. THEOREM. Let $X$ be a barrelled normed space, $\tau$ a locally convex topology of $X^{\prime}$ satisfying $(7)$ and $E=\left(X^{\prime}, \tau\right)$. quation

If $\left(t_{0}, x_{0}\right) \in \boldsymbol{R} \times E$ and $f: \boldsymbol{R} \times \mathbb{E} \rightarrow \mathbb{E}$ is continuous, the differential

$$
x^{\prime}(t)=f(t, x(t)), \quad x\left(t_{0}\right)=x_{0},
$$

has a local solution. In other words, $\mathbb{E}$ has the Peano property.

Proof. The theorem follows immediately from Theorems 3.1 and 3.2 and from Lemma 3.3.

3.5. Corollart (Peano). If $\left(t_{0}, x_{0}\right) \in \boldsymbol{R} \times \boldsymbol{R}^{n}$ and $f: \boldsymbol{R} \times \boldsymbol{R}^{n} \rightarrow \boldsymbol{R}^{n}$ is continuous, the differential equation

$$
x^{\prime}(t)=f(t, x(t)), \quad x\left(t_{0}\right)=x_{0},
$$

has a local solution.

Let $E=\left(X^{\prime}, \tau\right)$ be as in Theorem 3.4 and denote by $\|\cdot\|$ tho norm of $X^{\prime}$. If $\left(t_{0}, x_{0}\right) \in \boldsymbol{R} \times E$ and $f: \boldsymbol{R} \times E \rightarrow E$ is continuous, then by the Banach-Mackey theorem there is a real number $M, 1 \leqslant M<\infty$, such that

$$
\|f(t, x)\| \leqslant M \quad \text { if } \quad\left|t-t_{0}\right| \leqslant 1, \quad\left\|x-x_{0}\right\| \leqslant 1 .
$$

It is easy to see that every solution of (8) can be extended (if necessary) to the interval $I=\left[t_{0}, t_{0}+1 / M\right]$.

It is interesting to note that in the spaces of Theorem 3.4. also the Kneser's theorem holds:

3.6. Theorem. Let $E=\left(X^{\prime}, \tau\right),\left(t_{0}, x_{0}\right), f: \boldsymbol{R} \times E \rightarrow E$, $M$ and $I$ be as above. Then the set $S$ of all solutions $x: I \rightarrow E$ of the differential equation $(8)$ is compact and connected in $C(I, E)$. In partioular, the sets

$$
S(t)=\{x(t) \mid x \in S\}, \quad t \in I,
$$

are compact and connected subsets of $T$.

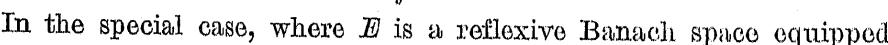
with the weak topology $\sigma\left(E, E^{\prime}\right)$, the theorem has been proved by szufla [12]. However, the method of Szufla applies equally well to the general case and therefore we shall omit the proof of Theorem 3.6 .

4. Semireflexivity and the Peano property. Prior to Godunov's papor [4] Cellina [1] proved that Peano's theorem cannot hold in any non reflexive Banach space (see also [2]). We shall generalize Cellina's con- struction to all quasi-complete locally corvex spaces that are not semireflexive. Also, a slight modification shows that Peano's theorem does not hold in any nonreflexive Banach space equipped with the weak topology. This gives a positive answer to the conjecture in [2].

The construction is based on the following

4.1. THEOREM (James, [8]). Let $E$ be a Banach space and $D$ a bounded and wealcly closed subset of $E$. If $D$ is not wealkly compact, there is a vector $v \in E^{\prime}$ such that

$$
\operatorname{sip}_{x \in D}\langle x, \nu\rangle=1 ; \quad\langle x, \nu\rangle<1 \quad \text { if } \quad x \in D .
$$

4.2. Coroltary. Let $E$ be a quasi-complete locally convex space and $D$ a closed, convex and bounded subset of $E$ which is not weakly compact. Then there is a vector $\nu \in E^{\prime}$ and a continuous function $g: E \rightarrow[0,1]$ such that

(a) $\sup \langle x, v\rangle=1$; $\langle x, \nu\rangle\langle 1$ if $x \in D$;

(b) $g(x)=1$ if $x \in D ; g(x)=0$ if $\langle x, v\rangle \geqslant 1$.

Proof. Let $\left\{q_{\alpha} \mid \alpha \in \mathscr{A}\right\}$ be a family of continuous seminorms generating the topology of $E$ and let $E_{a}, \alpha \in \mathscr{A}$, denote the completion of the quotient space $E / q_{\alpha}^{-1}(0)$. Then $E$ can be indentified with a subspace of the product $\prod_{a \in A} E_{\alpha}$ of the Banach spaces $E_{a}$ (see [9], p. 46). Denote by $P_{\beta}$ the cunonical projection $P_{\beta}: \prod_{\alpha \in \mathscr{A}} E_{\alpha} \rightarrow E_{\beta}, \beta \in \mathscr{A}$.

As $D$ is complete, $D$ is closed also in the product of the spaces $\boldsymbol{E}_{a}$, $a \in A$. Moreover, $\overline{P_{a}(D)}$ is a conrex, closed and bounded subset of $E_{a}$ for each $\alpha \in \mathscr{A}$. If $\overline{P_{\alpha}(D)}$ were weakly compact for every $\alpha \in \mathscr{A}$, the Tychonoff theorem and the Theorem 17.13 (iii) in [9] (p. 160) would imply the weak compactness (in $E$ ) of the set

$$
D \subset \prod_{a \in \mathscr{Q}} \overline{P_{a}(D)}
$$

Thus there must exist an index $\alpha \in \mathscr{A}$ such that $\overline{P_{\alpha}(D)}$ is not weakly compact in $E_{\alpha}$.

By Theorem 4.1. we can find a vector $\mu \in E_{\alpha}^{\prime}$ such that $\langle x, \mu\rangle<1$ for overy $x \in \overline{P_{a}(D)}$ even though $\sup \left\{\langle x, \mu\rangle \mid x \in \overline{P_{a}(D)}\right\}=1$. Now the olosed sets $P_{a}(D)$ and $G=\left\{x \in E_{a} \mid\langle x, \mu\rangle \geqslant 1\right\}$ are disjoint. As $E_{a}$ is normal, there is a continuous function $f: E_{\alpha} \rightarrow[0,1]$ such that $f(x)=0$ if $x \in G$ and $f(x)=1$ if $x \in P_{a}(D)$.

Finally, the mappings $\nu=\mu \odot P_{\alpha}$ and $g=f \circ P_{\alpha}$ satisfy the requirements (a) and (b).

4.3. Trworem. Let $E$ be a looally convex quasi-complete vector space. If $E$ is not semireflexive, there is a continuous mapping $f: \boldsymbol{R} \times E \rightarrow E$ such 
that the Cauchy problem

$$
x^{\prime}(t)=f(t, x(t)), \quad x(0)=\overline{0},
$$

admits no solution on any nonvanishing interval $[a, b]$ containing the origin.

Proof. As $E$ is not semireflexive, there is a weakly closed and bounded set $D \subset E$, which is not weakly compact ([7], p. 227). We can assume that $D$ is convex and balanced; then $D$ is closed also in the original topology of $E$.

Let $\nu \in E^{\prime}$ be a rector satisfying condition (a) of Corollary 4.2. Choose a sequence $\left\{x_{n}\right\}_{n=1}^{\infty} \subset D$ such that $0<\left\langle x_{n}, v\right\rangle\left\langle\left\langle x_{n+1}, v\right\rangle, n \in \boldsymbol{N}\right.$, and $\left\langle x_{n}, \nu\right\rangle \rightarrow 1$ as $n \rightarrow \infty$. Denote by $V$ the open set $V=\{x \in E \mid\langle x, \nu\rangle<1\}$. There is a partition of unity $\left\{q_{n}\right\}_{n=1}^{\infty}$ in $V$ subordinate to the corering $\left\{U_{n}\right\}_{n=1}^{\infty}$,

$$
\begin{aligned}
& U_{1}=\left\{x \in E \mid\langle x, v\rangle<2 \cdot\left\langle x_{2}, v\right\rangle-1\right\} \\
& U_{n}=\left\{x \in E \mid 2 \cdot\left\langle x_{n-1}, v\right\rangle-1<\langle x, v\rangle<2 \cdot\left\langle x_{n+1}, v\right\rangle-1\right\}, \quad n \geqslant 2,
\end{aligned}
$$

of $V$. This can be seen easily: Set

$$
\begin{aligned}
& W_{1}=\left\{s \in \boldsymbol{R} \mid s<2 \cdot\left\langle x_{2}, v\right\rangle-1\right\}, \\
& W_{n}=\left\{s \in \boldsymbol{R} \mid 2 \cdot\left\langle x_{n-1}, v\right\rangle-1<s<2 \cdot\left\langle x_{n+1}, v\right\rangle-1\right\}, \quad n \geqslant 2 .
\end{aligned}
$$

Then $\left\{W_{n}\right\}_{n=1}^{\infty}$ is an open covering of $(-\infty, 1)$. If $\left\{p_{n}\right\}_{n=1}^{\infty}$ is a partition of unity in $(-\infty, 1)$ subordinate to $\left\{W_{n}\right\}_{n=1}^{\infty}$, we may simply take $q_{n}=p_{n} \circ v$, $n \in \boldsymbol{N}$. Note that for each $x \in V, q_{n}(x)$ vanishes for all but at most two $n \in \boldsymbol{N}$.

The continuity of the mapping $F: V \rightarrow E$

$$
F(x)=\frac{1}{2}(\langle x, v\rangle+1) \sum_{n=1}^{\infty} q_{n}(x) x_{n+1} \mid\left\langle x_{n+1}, \nu\right\rangle
$$

is obvious. Let $p_{D}$ be the Minkowski functional of $D$ (we set $p_{D}(y)=\infty$, if $y \notin t D$ for any $t \in \boldsymbol{R})$. If $x \in V$, we choose an index $k \in \boldsymbol{N}$ such that $x \notin U_{n}$ if $n \neq k, k+1$. Then

$p_{D}\left(F^{\prime}(x)\right) \leqslant \frac{1}{2}|\langle x, \nu\rangle+1|\left\{q_{k}(x) /\left\langle x_{k+1}, \nu\right\rangle+q_{k+1}(x) /\left\langle x_{k+2}, v\right\rangle\right\}$

$$
\leqslant \frac{1}{2}\left\{q_{k}(x) 2\left\langle x_{k+1}, v\right\rangle \mid\left\langle x_{k+1}, v\right\rangle+q_{k+1}(x) 2\left\langle x_{k+2}, v\right\rangle /\left\langle x_{k++2}, v\right\rangle\right\}=1 \text {. }
$$

In other words $F(V) \subset D$. Moreover,

$$
\langle F(x), \nu\rangle=\frac{1}{2}(\langle x, \nu\rangle+1) \sum_{n=1}^{\infty} q_{n}(x)=\frac{1}{2}(\langle x, \nu\rangle+1) .
$$

Let $g: E \rightarrow[0,1]$ be a continuous mapping having properties (b) of Corollary 4.2. We set

$$
G(x)= \begin{cases}\frac{g}{0}(x) F(x), & x \in V \\ 0, & x \in E \backslash V .\end{cases}
$$

Because $F$ is bounded, $G$ is continuous in the whole space $E$. In addition $\left.F\right|_{D}=\left.G\right|_{D}$. Finally, we define

$$
f(t, x)= \begin{cases}2 t G\left(x / t^{2}\right), & x \in E, t \neq 0, \\ 0, & x \in E, t=0 .\end{cases}
$$

By the boundedness of $G$ f: $\boldsymbol{R} \times E \rightarrow E$ is continuous.

Let us now assume that $x:[0, \delta] \rightarrow E, \delta>0$, is a solution of the differential equation (9). First, we claim that

$$
x(t) / t^{2} \in D, \quad t \in(0, \delta] .
$$

In fact, by the separation form of the Hahn-Banach theorem $D$ is the intersection of all the barrelled neighbourhoods of $\overline{0}$ containing $D$. Therefore it suffices to show that $x(t) / t^{2} \in U$ whenever $U$ is a barrelled neighbourhood of $\ddot{0}$ and $D \subset U$.

If $p_{U}$ denotes the Minkowski functional of $U, p_{U}\left(G\left(x / t^{2}\right)\right) \leqslant 1$ as $G\left(x / t^{2}\right) \in D \subset U$. Since $p_{U}$ is a continuous seminorm, this yields

$$
p_{U}(x(t)) \leqslant \int_{0}^{t} p_{U}(f(s, x(s))) d s \leqslant \int_{0}^{t} 2 s d s=t^{2},
$$

which proves formula (11). Further, as $x(t) / t^{2} \in D, f(t, x(t))=2 t F\left(x(t) / t^{2}\right)$. Now, by $(10)$,

which leads to

$$
\begin{aligned}
d / d t\langle x(t), v\rangle= & 2 t\left\langle F\left(x(t) \mid t^{2}\right), v\right\rangle \\
& =2 t \cdot \frac{1}{2}\left(\left\langle x(t) \mid t^{2}, v\right\rangle+1\right)=\frac{1}{t}\langle x(t), v\rangle+t,
\end{aligned}
$$

$$
\frac{d}{d t}\left[\frac{\langle x(t), v\rangle}{t}\right]=\frac{t\left\langle x^{\prime}(t), v\right\rangle-\langle x(t), v\rangle}{t^{2}}=1 .
$$

Since $\langle x(t), v\rangle \mid \leqslant t^{2}$, we get $\langle x(t), v\rangle=t^{2}$ or $\left\langle x(t) / t^{2}, v\right\rangle=1$. This, however, is in contradiction with formula (11) and Corollary 4.2(a).

4.4. THeorem. Let $E$ be a Banach space. Then $E$ is reflexive if and only if the space $\left(\mathbb{E}, \sigma\left(\mathbb{E}, \mathbb{E}^{\prime}\right)\right)$ has the Peano property.

Proof. The "only if"-part follows from Theorem 3.4 (or from [11]). To show the converse it sulfices by the proof of Theorem 4.3 to find for every nonretlexive Banach space $E$ a vector $\nu \in \mathbb{E}^{\prime}$ and a wealkly continuous function $g: E \rightarrow[0,1]$ such that

$$
\begin{aligned}
& \text { (12.a) }\|v\|=1 ; \quad\langle x, v\rangle<1 \quad \text { if }\|x\| \leqslant 1 ; \\
& \text { (12.b) } \quad g(x)=1 \quad \text { if }\|x\| \leqslant 1 ; \quad g(x)=0 \quad \text { if }\langle x, v\rangle \geqslant 1 .
\end{aligned}
$$

Now, let $E$ be a nonreflexive Banach space. If $M$ is a closed, separable and nonreflexire subspace of $E$, then there exists a norming sequence 
$\left\{x_{n}^{\prime}\right\}_{n=1}^{\infty}$ in the closed unit ball of $\mathbb{M}{ }^{\prime}$, i.e.

$$
\sup _{n \in \boldsymbol{N}}\left|\left\langle x, x_{n}^{\prime}\right\rangle\right|=\|x\| \quad \text { for every } x \in M .
$$

Also, the linear mapping $T: E \rightarrow l^{\infty}, x \mapsto\left(\left\langle x, x_{n}^{\prime}\right\rangle\right)_{n=1}^{\infty}$ is continuous; in fact, $\|T\|=1$.

Let $B_{M}$ and $B_{E}$ stand for the closed unit bulls of the Banach spaces $M$ and $E$, respectively. Since the restriction of $T$ to $M$ is an isometry (cf. (13)), $T\left(B_{M}\right)$ is closed and convex but not weakly compact in $l^{\infty}$ Denote by $N$ the closure of $T E$ and by $D$ the closure of $T B_{H}$ in $l^{\infty}$. As $T\left(B_{M}\right) \subset N, N$ is a closed nonreflexivo subspace of $l^{\infty}$ having $D$ as its closed unit ball. Thus we may apply James' theorem and obtain a norm-one vector $\mu \in N^{\prime}$ that does not attain its maxinum on $D$.

The definition

$$
h: N \rightarrow \boldsymbol{R}, \quad x \mapsto \sup \{0,1-\langle x, \mu\rangle\}
$$

gives a weakly continuous mapping $h$ that vanishes exactly on the set $\{x \in N \mid\langle x, \mu\rangle \geqslant 1\}$. Moreover, let $\alpha_{n}$ stand for the $n$th coordinate function $\alpha_{n}:\left(x_{k}\right)_{k=1}^{\infty} \mapsto x_{n}$ and write $\beta_{n}(x)=\sup \left\{\left|\left\langle x, \alpha_{n}\right\rangle\right|-1,0\right\}, x \in N$.

Obviously $D$ equals the intersection of the sets $\beta_{n}^{-1}(0), n=1,2,3, \ldots$ Then, according to [3], 1.14 (a) and [3], Theorem 1.15, there exists a weakly continuous mapping $\tilde{g}: N \rightarrow[0,1]$ such that $\tilde{g}(x)=0$ if $\langle x, \mu\rangle \geqslant 1$, and $\tilde{g}(x)=1$ if $x \in D$. It is immediately seen that the mappings $g$ and $\nu$,

$$
g=\tilde{g} \circ T: E \rightarrow[0,1] ; \quad \nu=T^{\prime} \mu \in E^{\prime}
$$

satisfy conditions (12).

Acknowledgments. The author wishes to express his gratitude to J. Luukkainen for his kind advice related to Theorem 4.4.

\section{References}

[1] A. Cellina, On the nonexistence of solutions of differential errutions in nonreflexive spaces, Bull. Amer. Math. Soc. 78 (1972), 1069-1072.

[2] G.D. Faulkner, On the nonexistence of weato solutions to abstrat differential equations in nonreflexive spaces, Nonlinear Analysis 2 (1978), 505-508.

[3] L. Gillm an and M. Jorison, Rings of continuous functions, Springer-Verlag, Now York, Heidelberg, Berlin 1976. [4] A. N. Godunov, Peano's theorem in Banach spaces, Functional. Anal. Appl.
9 (1975), 53-55.

[5] A. Grothondieck, Topological vector spaces, Gordon and Brench, Now York 1975.

[0] P. Hartman, Ordinary differential equations, Jolnn Wiley\& Sons, Now York

[7] J. Horváth, Topological vector spaces and distributions, Addison-Wesley, 966.
[8] R. C. James, Wealcly compuct sets, Trans. Amer. Math. Soc. 113 (1964), 129-140. [9] J.L. Kelley and I. Namioka, Linear topological spaces, Van Nostrand, Princetion 1963.

[10] S. Rolewicz, Metrio linear spaces, Monografie Matematyczne, Warsaw 1972.

[11] A. Szóp, Existence theorem for weak solutions of ordinary differential equations in reflexive Banasih spcces, Studia Sci. Math. Hungar. 6 (1971), 197-203.

[12] S. Szufla, Kneser's theorem for weate solutions of ordinary differential equations in reflexive Banach spaces, Bull. Acad. Polon. Sci. Sór. Sci. Math. Astronom. Phys. 26 (1078), 407-4.13. 